\title{
Parameter identification for multiple modes of cable-stayed bridge cables using ambient vibration measurements from a single station
}

\author{
W.-H. Wu, C.-A. Liao \& C.-C. Chen \\ Department of Construction Engineering, \\ National Yunlin University of Science and Technology, Taiwan
}

\begin{abstract}
The measurements and subsequent system identification of the cables play extremely important roles in health monitoring of the whole cable-stayed bridge. The technique of ambient vibration measurement where only the output signal is available has been commonly adopted to measure the cable-stayed bridges. In this case, it is most popular in the literature to combine the random decrement method together with the Ibrahim time domain method for system identification. To apply the above two methods for cable identification, however, the problem of imperfect random decrement signatures and the difficulty of conducting well-distributed measurements at various stations of a single cable have to be overcome. The crucial time shifting parameter is first explored in this study to extend the applicability of the Ibrahim time domain method. In addition, with the mode separation technique and a novel multiple random decrement method recently proposed, an effective method to identify the parameters of several cable modes merely based on the measurement of a single station is developed and demonstrated by applying it to the velocity record of a cable of the Chi- $\mathrm{Lu}$ cable-stayed bridge. The validation of this method is also provided in this paper.

Keywords: ambient vibration measurement, Ibrahim time domain method, time shifting, mode separation, multiple random decrement method.
\end{abstract}

\section{Introduction}

The cables of cable-stayed bridges are the primary force-transmitting members of the whole structure system. Consequently, the measurements and subsequent system identification of the cables play extremely important roles in health 
monitoring of the whole cable-stayed bridge. The dynamic response of the cable usually attributes to quite a few of the lower modes due to its low flexural stiffness. It is accordingly required to obtain the modal parameters of more modes in the identification of cable than other civil structures.

With the advantages of mobility and easy setup, the technique of ambient vibration measurement has been recently applied to conduct the system identification for most of the cable-stayed bridges. Since only the output signal is available in this case, it is necessary to apply the system identification techniques based merely on output signals for determining the modal parameters of cables. Limited by in situ working constraints, it is usually difficult to conduct the measurement with multiple stations uniformly distributed along the same cable. Moreover, the insignificant signals from ambient vibration also induce problems such as noise pollution in practical applications.

To tackle the above problems, it is aimed in this study to develop an effective method for accurately identifying the parameters of various cable modes merely based on the measurement of a single station installed on cables. For identifying the modal parameters simply based on output signals, it is most popular in the literature to combine the random decrement (RD) method together with the Ibrahim time domain (ITD) method. To apply the above two methods for cable parameter identification, however, two major problems need to be overcome. First of all, imperfect RD signatures may be usually induced from the cable measurements due to narrow-banded excitations. Furthermore, the ITD method is not valid to identify the parameters for multiple modes when only the measurement of a single station is available. The crucial time shifting parameter is first explored in this study to extend the applicability of the Ibrahim time domain method. In addition, with the mode separation technique and a novel multiple random decrement method recently proposed, an effective method to identify the modal parameters of cable is developed. Demonstrated by applying it to the measured velocity records of the cables of Chi-Lu cable-stayed bridge, the validation of this method is also provided in this paper.

\section{Random decrement and Ibrahim time domain methods}

In this section, the RD and ITD methods will be briefly reviewed to clarify a few problems for directly applying them in the modal parameter identification of cables, which provides the foundation for developing a novel and more effective identification method.

\subsection{Random decrement method}

Assume that a linear system with $n$ degrees-of-freedom is subjected to a stationary white noise with a zero mean. The corresponding equations of motion can then be expressed as:

$$
\mathbf{M} \ddot{\mathbf{x}}(t)+\mathbf{C} \dot{\mathbf{x}}(t)+\mathbf{K x}(t)=\mathbf{f}(t)
$$


where $\mathbf{M}, \mathbf{C}$, and $\mathbf{K}$ represent the $n \times n$ structural mass, damping, and stiffness matrix, respectively. In addition, $\mathbf{x}(t)$ signifies the displacement response vector and $\mathbf{f}(t)$ is the excitation force vector. Considering that Equation (1) is satisfied by this system at instant $t_{i}$, a time shifting of $\tau$ leads to:

$$
\mathbf{M} \ddot{\mathbf{x}}\left(t_{i}+\tau\right)+\mathbf{C} \dot{\mathbf{x}}\left(t_{i}+\tau\right)+\mathbf{K x}\left(t_{i}+\tau\right)=\mathbf{f}\left(t_{i}+\tau\right)
$$

Selecting $N$ different starting instants from eqn (2) and then computing their mean, it yields:

$$
\mathbf{M} \ddot{\mathbf{y}}(\tau)+\mathbf{C} \dot{\mathbf{y}}(\tau)+\mathbf{K y}(\tau)=\frac{1}{N} \sum_{i=1}^{N} \mathbf{f}\left(t_{i}+\tau\right)=\mathbf{0}_{n \times 1} \text { as } N \rightarrow \infty
$$

where $\mathbf{y}(\tau)=\left[\sum_{i=1}^{N} \mathbf{x}\left(t_{i}+\tau\right)\right] / N$ and $\mathbf{0}$ stands for a zero matrix with its dimension indicated in the subscript. Since $\mathbf{f}(t)$ is assumed to be a zero-mean and stationary white noise, the right-hand side of eqn (3) has to become a zero vector when $N \rightarrow \infty$. Consequently, eqn (1) describing the forced vibration is now turned into another free vibration equation in the form of eqn (3) where the variable vector $\mathbf{x}(t)$ is replaced by $\mathbf{y}(\tau)$. In general, a stable characteristic function $\mathbf{y}(\tau)$, usually called the random decrement signature in the literature, can be obtained when $N>500$ (Cole [1]). It was also pointed out by other studies that $N=1000$ is the optimal number of superposition and $N=100$ is the minimum acceptable number (Jeary [2]).

Taking a single velocity signal $\dot{x}(t)$ for example, its random decrement signature can be obtained with the following detailed steps:

1. Choose a fixed value of velocity $\dot{x}_{s}$ as the cutting threshold such that $\dot{x}(t)$ is with values of $\dot{x}_{s}$ at $N$ different time instants $t_{1}, t_{2}, \cdots \cdots, t_{N}$.

2. Set the extracted signal duration $T_{d}$ such that the extracted signal can adequately reflect the dynamic characteristics of system.

3. Extract $N$ different time histories, all with a duration $T_{d}$, from the measured signal. Average all those time histories to yield the corresponding random decrement signature $\dot{y}(\tau)$.

\subsection{Ibrahim time domain method}

If the random decrement signature can be obtained from the ambient vibration measurements of cables and sufficiently represents the corresponding free vibration response, the ITD method is then usually applied to determine the associated modal parameters. Consider the free vibration system illustrated in eqn (3), but replace the time variable $\tau$ by $t$. Transformation of this equation into the state space further results in a first-order differential equation: 


$$
\left\{\begin{array}{c}
\dot{\mathbf{y}} \\
\ddot{\mathbf{y}}
\end{array}\right\}=\left[\begin{array}{cc}
\mathbf{0}_{n \times n} & \mathbf{I}_{n \times n} \\
-\mathbf{M}^{-1} \mathbf{K} & -\mathbf{M}^{-1} \mathbf{C}
\end{array}\right]\left\{\begin{array}{c}
\mathbf{y} \\
\dot{\mathbf{y}}
\end{array}\right\}
$$

where I indicates an identity matrix with its dimension shown in the subscript. Solving eqn (4) as an eigenvalue problem, the displacement and velocity vectors can be expressed, respectively as:

$$
\mathbf{y}(t)=\sum_{i=1}^{2 n} \boldsymbol{\Gamma}_{i} e^{\lambda_{i} t} ; \dot{\mathbf{y}}(t)=\sum_{i=1}^{2 n} \lambda_{i} \boldsymbol{\Gamma}_{i} e^{\lambda_{i} t}=\sum_{i=1}^{2 n} \boldsymbol{\Phi}_{i} e^{\lambda_{i} t}
$$

where $\lambda_{i}$ 's are the eigenvalues of the system matrix and $\boldsymbol{\Gamma}_{i}$ 's are the corresponding $n \times 1$ eigenvectors.

Based on eqn (5), the velocity vectors at $s$ different time instants can be assembled into a matrix as

$$
\left[\begin{array}{llll}
\dot{\mathbf{y}}_{1} & \dot{\mathbf{y}}_{2} & \cdots & \dot{\mathbf{y}}_{s}
\end{array}\right]=\left[\begin{array}{llll}
\boldsymbol{\Phi}_{1} & \boldsymbol{\Phi}_{2} & \cdots & \boldsymbol{\Phi}_{2 n}
\end{array}\right]\left[\begin{array}{cccc}
e^{\lambda_{1} t_{1}} & e^{\lambda_{1} t_{2}} & \cdots & e^{\lambda_{1} t_{s}} \\
e^{\lambda_{2} t_{1}} & e^{\lambda_{2} t_{2}} & \cdots & e^{\lambda_{2} t_{s}} \\
\vdots & \vdots & \ddots & \vdots \\
e^{\lambda_{2 n} t_{1}} & e^{\lambda_{2 n} t_{2}} & \cdots & e^{\lambda_{2 n} t_{s}}
\end{array}\right]
$$

where $\dot{\mathbf{y}}_{i}=\dot{\mathbf{y}}\left(t_{i}\right), i=1,2, \cdots, s$. If eqn (6) is shifted forward in the time axis with $m \Delta t$ and $2 m \Delta t$, respectively, then

$$
\begin{aligned}
& \overline{\mathbf{Y}}=\left[\begin{array}{llll}
\dot{\mathbf{y}}_{1} & \dot{\mathbf{y}}_{2} & \cdots & \dot{\mathbf{y}}_{s}
\end{array}\right]=\left[\begin{array}{llll}
\overline{\boldsymbol{\Phi}}_{1} & \overline{\boldsymbol{\Phi}}_{2} & \cdots & \overline{\boldsymbol{\Phi}}_{2 n}
\end{array}\right]\left[\begin{array}{cccc}
e^{\lambda_{1} t_{1}} & e^{\lambda_{1} t_{2}} & \cdots & e^{\lambda_{1} t_{s}} \\
e^{\lambda_{2} t_{1}} & e^{\lambda_{2} t_{2}} & \cdots & e^{\lambda_{2} t_{s}} \\
\vdots & \vdots & \ddots & \vdots \\
e^{\lambda_{2 n} t_{1}} & e^{\lambda_{2 n} t_{2}} & \cdots & e^{\lambda_{2 n} t_{s}}
\end{array}\right]=\overline{\boldsymbol{\Phi}} \boldsymbol{\Lambda}
\end{aligned}
$$

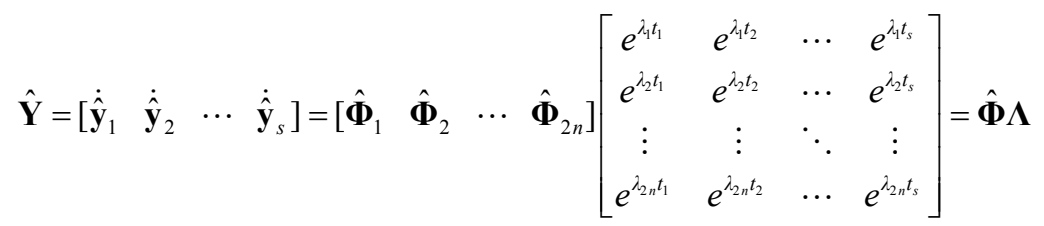

where $\quad \dot{\overline{\mathbf{y}}}_{i}=\dot{\mathbf{y}}\left(t_{i}+m \Delta t\right)$ and $\quad \dot{\hat{\mathbf{y}}}_{i}=\dot{\mathbf{y}}\left(t_{i}+2 m \Delta t\right), i=1,2, \cdots, s . \quad$ In addition, $\overline{\boldsymbol{\Phi}}_{i}=\boldsymbol{\Phi}_{i} e^{\lambda_{i} m \Delta t}$ and $\hat{\boldsymbol{\Phi}}_{i}=\boldsymbol{\Phi}_{i} e^{\lambda_{i} 2 m \Delta t}, i=1,2, \cdots, 2 n$. Two combinations of eqns (6)-(8) can be obtained as

$$
\mathbf{U}=\left[\begin{array}{l}
\mathbf{Y} \\
\overline{\mathbf{Y}}
\end{array}\right]=\left[\begin{array}{l}
\boldsymbol{\Phi} \\
\overline{\boldsymbol{\Phi}}
\end{array}\right] \boldsymbol{\Lambda}=\overline{\mathbf{\Psi}} \boldsymbol{\Lambda} \text { and } \mathbf{V}=\left[\begin{array}{c}
\overline{\mathbf{Y}} \\
\hat{\mathbf{Y}}
\end{array}\right]=\left[\begin{array}{c}
\overline{\boldsymbol{\Phi}} \\
\hat{\mathbf{\Phi}}
\end{array}\right] \boldsymbol{\Lambda}=\hat{\mathbf{\Psi}} \boldsymbol{\Lambda}
$$

where $\mathbf{U}$ and $\mathbf{V}$ are $2 n \times s$ matrices and $\overline{\boldsymbol{\Psi}}$ and $\hat{\boldsymbol{\Psi}}$ are $2 n \times 2 n$ matrices. Examination of eqn (9) reveals that if a system related matrix $\mathbf{A}$ is defined as 


$$
\mathbf{A} \overline{\boldsymbol{\Psi}}=\hat{\boldsymbol{\Psi}}=\overline{\mathbf{\Psi}}\left[\begin{array}{cccc}
e^{\lambda_{1} m \Delta t} & 0 & \cdots & 0 \\
0 & \ddots & \vdots & \vdots \\
\vdots & \vdots & \ddots & 0 \\
0 & \cdots & 0 & e^{\lambda_{2 n} m \Delta t}
\end{array}\right]
$$

then eqn (10) obviously indicates that $e^{\lambda_{1} m \Delta t}, \cdots, e^{\lambda_{2 n} m \Delta t}$ are the eigenvalues of $\mathbf{A}$ and directly related to the system eigenvalues: $\lambda_{1}, \cdots, \lambda_{2 n}$. Pre-multiplying the first part in eqn (9) by $\mathbf{A}$ and applying eqn (10) lead to:

$$
\mathbf{A U}=\mathbf{A} \overline{\mathbf{\Psi}} \boldsymbol{\Lambda}=\hat{\mathbf{\Psi}} \boldsymbol{\Lambda}=\mathbf{V}
$$

Therefore, the least squares method can be conveniently adopted to obtain $\mathbf{A}$ based on eqn (11), followed by the previously mentioned eigenvalue analysis.

It has been shown in the literature that the eigenvalues of the state space system in eqn (4) have to be in complex conjugate pairs and the eigenvalues $\lambda_{2 j-1}$ and $\lambda_{2 j}$ corresponding to the $j$-th mode are related to the modal frequency $\omega_{j}$ and modal damping ratio $\xi_{j}$ by

$$
\lambda_{2 j-1}, \lambda_{2 j}=-\xi_{j} \omega_{j} \pm \mathrm{i} \omega_{j} \sqrt{1-\xi_{j}^{2}}=\alpha_{j} \pm \mathrm{i} \beta_{j}, j=1,2, \cdots, n
$$

Consequently, the eigenvalues of $\mathbf{A}$ also appear in complex conjugate pairs as

$$
\begin{aligned}
e^{\lambda_{2 j-1} m \Delta t}, e^{\lambda_{2 j} m \Delta t} & =e^{\alpha_{j} m \Delta t}\left[\cos \left(\beta_{j} m \Delta t\right) \pm \mathrm{i} \sin \left(\beta_{j} m \Delta t\right)\right] \\
& =a_{j} \pm \mathrm{i} b_{j}, j=1,2, \cdots, n
\end{aligned}
$$

With eqn (13), it is easy to obtain:

$$
\alpha_{j}=\frac{1}{2 m \Delta t} \ln \left(a_{j}^{2}+b_{j}^{2}\right) \text { and } \beta_{j}=\frac{1}{m \Delta t} \tan ^{-1}\left(\frac{b_{j}}{a_{j}}\right), j=1,2, \cdots, n
$$

and then yield:

$$
\omega_{j}=\left|\lambda_{2 j-1}\right|=\left|\lambda_{2 j}\right|=\sqrt{\alpha_{j}^{2}+\beta_{j}^{2}} \text { and } \xi_{j}=\frac{-\alpha_{j}}{\omega_{j}}, j=1,2, \cdots, n
$$

\section{Parametric study for time shifting in the ITD method}

The combination of RD and ITD methods, as described in the previous section, can usually provide meaningful results for several modal parameters if the ambient vibration measurements can be obtained for various stations of the target system and the frequency content of environmental excitations is not far away from that of a white noise. Unfortunately, the above two conditions are not always possible for certain civil structures, especially the cable-stayed bridge 
cables. To overcome these critical difficulties in practical applications, a first step is to improve the accuracy of the ITD method if the input time history is not an ideally decaying function under free vibration. As described in Section 2.2, the matrix A utilized in the ITD method has to be constructed by shifting a certain amount of time and its eigenvalues are then solved to determine the modal frequencies and damping ratios of system. It is obvious that different adopted values for the number of shifted time steps $m$ will certainly lead to different matrices of $\mathbf{A}$ and altered eigenvalues, especially for the case with a response not closed related to a free vibration function. The parametric study of $m$ is consequently conducted in this section to determine the optimal selection.

Table 1: Identified parameters of SDOF systems for various time shifting values.

\begin{tabular}{|c|c|c|c|c|c|c|}
\hline \multirow[b]{2}{*}{$\begin{array}{l}\text { No. of shifted } \\
\text { time steps } \\
\quad m\end{array}$} & \multicolumn{3}{|c|}{ SDOF system with damping ratio $0.1 \%$} & \multicolumn{3}{|c|}{ SDOF system with damping ratio $1 \%$} \\
\hline & $\begin{array}{l}\text { Eigenvalues } \\
\text { of } \mathbf{A}\end{array}$ & \begin{tabular}{|c} 
Natural \\
Frequency \\
$\omega(\mathrm{Hz})$
\end{tabular} & $\begin{array}{c}\text { Damping } \\
\text { Ratio } \\
\xi(\%)\end{array}$ & $\begin{array}{c}\text { Eigenvalues } \\
\text { of } \mathbf{A}\end{array}$ & \begin{tabular}{|c|} 
Natural \\
Frequency \\
$\omega(\mathrm{Hz})$
\end{tabular} & $\begin{array}{c}\text { Damping } \\
\text { Ratio } \\
\xi(\%)\end{array}$ \\
\hline 5 & $0.987 \pm 0.156 \mathrm{i}$ & 0.9999 & 1.000 & $0.965 \pm 0.155 \mathrm{i}$ & 1.0259 & 14.006 \\
\hline 10 & $0.951 \pm 0.309 \mathrm{i}$ & 0.9997 & 0.103 & $0.930 \pm 0.306 \mathrm{i}$ & 1.0149 & 6.719 \\
\hline 15 & $0.891 \pm 0.454 \mathrm{i}$ & 0.9997 & 0.067 & $0.873 \pm 0.450 \mathrm{i}$ & 1.0096 & 3.736 \\
\hline 20 & $0.809 \pm 0.587 \mathrm{i}$ & 0.9996 & 0.047 & $0.799 \pm 0.584 \mathrm{i}$ & 1.0056 & 1.650 \\
\hline 25 & $0.707 \pm 0.707 i$ & 0.9996 & 0.038 & $0.702 \pm 0.705 \mathrm{i}$ & 1.0027 & 0.724 \\
\hline 30 & $0.588 \pm 0.809 \mathrm{i}$ & 0.9996 & 0.036 & $0.584 \pm 0.806 \mathrm{i}$ & 1.0010 & 0.488 \\
\hline 35 & $0.454 \pm 0.890 \mathrm{i}$ & 0.9996 & 0.036 & $0.451 \pm 0.887 \mathrm{i}$ & 1.0005 & 0.453 \\
\hline 40 & $0.309 \pm 0.950 \mathrm{i}$ & 0.9996 & 0.036 & $0.307 \pm 0.946 \mathrm{i}$ & 1.0005 & 0.436 \\
\hline 45 & $0.157 \pm 0.987 \mathrm{i}$ & 0.9996 & 0.036 & $0.155 \pm 0.982 \mathrm{i}$ & 1.0006 & 0.430 \\
\hline 50 & $0.001 \pm 0.999 \mathrm{i}$ & 0.9996 & 0.0 & $-0.001 \pm 0.993 \mathrm{i}$ & 0.9994 & 0.427 \\
\hline 55 & $-0.156 \pm 0.987 \mathrm{i}$ & 0.9996 & 0.036 & $-0.156 \pm 0.980 \mathrm{i}$ & 1.0004 & 0.418 \\
\hline 60 & $-0.308 \pm 0.951 \mathrm{i}$ & 0.9996 & 0.036 & $-0.307 \pm 0.944 \mathrm{i}$ & 1.0001 & 0.419 \\
\hline 65 & $-0.453 \pm 0.891 \mathrm{i}$ & 0.9996 & 0.036 & $-0.449 \pm 0.883 \mathrm{i}$ & 0.9997 & 0.450 \\
\hline 70 & $-0.586 \pm 0.809 \mathrm{i}$ & 0.9996 & 0.036 & $-0.581 \pm 0.801 \mathrm{i}$ & 0.9995 & 0.496 \\
\hline 75 & $-0.706 \pm 0.707 \mathrm{i}$ & 0.9996 & 0.036 & $-0.697 \pm 0.699 \mathrm{i}$ & 0.9993 & 0.535 \\
\hline 80 & $-0.808 \pm 0.588 \mathrm{i}$ & 0.9996 & 0.037 & $-0.796 \pm 0.581 \mathrm{i}$ & 0.9991 & 0.588 \\
\hline 85 & $-0.890 \pm 0.455 \mathrm{i}$ & 0.9996 & 0.038 & $-0.872 \pm 0.448 \mathrm{i}$ & 0.9988 & 0.734 \\
\hline 90 & $-0.950 \pm 0.310 \mathrm{i}$ & 0.9996 & 0.041 & $-0.919 \pm 0.303 \mathrm{i}$ & 0.9984 & 1.170 \\
\hline 95 & $-0.986 \pm 0.157 \mathrm{i}$ & 0.9996 & 0.062 & $-0.889 \pm 0.124 \mathrm{i}$ & 1.0069 & 3.596 \\
\hline 100 & $0.236 ;-0.988$ & $\cdot$ & $\cdot$ & $0.760 ;-0.999$ & $\cdot$ & $\cdot$ \\
\hline 150 & $-0.002 \pm 0.998 \mathrm{i}$ & 0.9996 & 0.035 & $0.001 \pm 0.981 \mathrm{i}$ & 1.0002 & 0.414 \\
\hline 200 & $0.189 ; 0.973$ & $\cdot$ & $\cdot$ & $0.535 ; 0.998$ & $\cdot$ & $\cdot$ \\
\hline
\end{tabular}

The simulated response functions for a single-degree-of-freedom (SDOF) system with various parameter values (natural frequency $\omega=1 \mathrm{~Hz}$ and damping ratio $\xi=0.1,1 \%$ ) are adopted to investigate the effects of the time shifting parameter in the ITD method. For these two different cases, the same initial displacement and ground acceleration reflecting the measured environmental 
excitation are assumed to compute the corresponding displacement time histories of 300 seconds. A threshold of $80 \%$ of the standard deviation of each time history and an extracted duration of $T_{d}=150 \mathrm{sec}$ are adopted to carry out the RD method. Those selected values for the RD parameters do not correspond to the optimal choice (Liao [3]) and are intended to result in the imperfect RD signatures where the excitation effects are not totally filtered out. In addition, the examined values of $m$ are limited to the time shifting range within a period of system, especially focused on the shifting less than a half of the period. It should be noted that $m=200$ corresponds to a time shifting of a period since $\Delta t=0.005 \mathrm{sec}$ is taken in computation. The identified frequencies and damping ratios from the ITD method for all these different cases are listed in Table 1.

From the results in Table 1, it is evidently observed that the identified natural frequencies and damping ratios from the ITD method converge to stable values as the shifting parameter $m$ gradually increases from small values, especially when the time shifting reaches one quarter of the system period $(m=50)$. Nevertheless, the identified results would get worse if $m$ keeps increasing to larger values. The worse case is bumped as the time shifting grows up to a half of the system period ( $m=100$ ) where real eigenvalues of $\mathbf{A}$ are obtained and no feasible system parameters can be identified. The above comparison clearly indicates that the value of $m$ corresponding to a shifting of one quarter of the system period is the optimal selection while the value of $m$ corresponding to a shifting of a half of the system period needs to be always avoided.

The reason why the shifting parameter $m$ is capable of inducing such a critical difference can be explained by considering the periodic characteristics for the free vibration of SDOF systems. If the ITD method is applied on an ideal time history of free vibration, the time shifting of one half of the system period would only find another time history that is different from the original time history in signs and with a scalar factor. Thus, the associated matrix $\mathbf{A}$ will not be full-ranked and it is impossible to solve for sufficient eigenvalues. In a more general sense, the same problem will be encountered if the time shifting is taken as a multiple of one half of the system period. Theoretically, the other values of time shifting are eligible for effective identifications. When the RD signature is not an ideal time history of free vibration, however, different values of time shifting in the application of the ITD method will include deviated errors into $\mathbf{A}$ and produce the identified results with a variety of accuracy. As the time shifting is close to one quarter of the system period, the least related original and shifted time histories will be obtained and consequently lead to the optimal identification. Similarly, the values of time shifting such as three or five quarters of the system period will also yield pretty good results. As for the values of time shifting close to a multiple of one half of the system period, the shifted time history will be nearly dependent on the original time history such that real eigenvalues of $\mathbf{A}$ will be obtained and no feasible identification is possible. It is also noteworthy that the identified natural frequencies in different cases are all in excellent accuracy while the errors for the identified damping ratios may not be negligible. This problem comes from the fact that the RD method cannot totally exclude the effects of external excitations and will be next discussed. 


\section{Multiple random decrement method}

In a very recent study by the authors (Wu [4]), a novel multiple random decrement (MRD) method was proposed to successfully exclude the effects from background excitation. It will be briefly reviewed and illustrate in this section for developing an effective method to identify the modal parameters of cable.

The ambient vibration measurement for a cable numbered L33 (length $=$ $126.4 \mathrm{~m}$ and inclined angle $=26^{\circ}$ ) of the Chi-Lu cable-stayed bridge close to the epicenter of 1999 Ch-Chi earthquake occurred in Taiwan can be taken as an example for illustration. In Figures 1 and 2, respectively, the originally measured velocity signal and its corresponding $\mathrm{RD}$ signature (cutting threshold $=0.8 \times$ standard deviation of the original measurement and $T_{d}=$ half of the original time history) are displayed in both forms of time history and Fourier amplitude spectrum. It is noteworthy in Figure 1 that all the cable frequencies are nearly in an arithmetic sequence, as predicted by the string theory. However, there exist two additional peak frequencies. These two extra peak frequencies have been pointed out to be corresponding to two significant modal frequencies of the bridge deck (Liao [3]). From the cable's standpoint, these two frequencies can be regarded as the particularly concentrated parts in the frequency content of external excitation. Even in this case where the excitation is far away from a white noise, Figure 2 indicates that the contributions from these two non-cable frequencies have been considerably suppressed after conducting the RD method. Therefore, in the cases where the excitation force is not close to a white noise, the RD method can still greatly raise the contributions from all the modal frequencies and also relatively diminish those from the rest frequencies even though the resulted RD signature may not be a perfect free vibration time history. Based on this generalized concept, a novel multiple random decrement (MRD) method was proposed. More specifically, the conventional RD method can be repeatedly applied on each round of the resulted RD signature to exclusively filter out the initially substantial effects of the excitation frequencies such that the goal of extracting the free vibration time history can be practically attained.
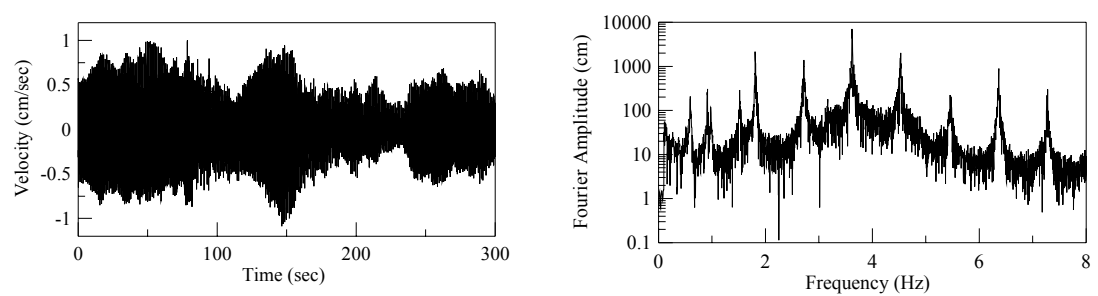

Figure 1: $\quad$ Original measurement for Cable 33 of the Chi-Lu bridge.

Two associated problems have to be solved before the MRD can be effectively applied in the identification of cable parameters. First of all, the extracted duration $T_{d}$ in each round of $\mathrm{RD}$ is the length of time history for the 
next round and thus needs to be taken as large as possible such that a sufficient superposition number $N$ can be reached in the next round. But contradictorily, no adequate number $N$ would be attained if a very large value of $T_{d}$ is selected in the current round. To balance this problem for mutually compensated $N$ and $T_{d}$ in each round of $\mathrm{RD}$, it was suggested (Wu [4]) to choose $T_{d}$ as a half length of the signal from the previous round. As for the second problem, the associated characteristic of the MRD method to also suppress the contributions from the secondary modes makes it impossible for the subsequent ITD identification to directly obtain meaningful parameters for several modes at one time. This obstacle will be overcome in the next section.
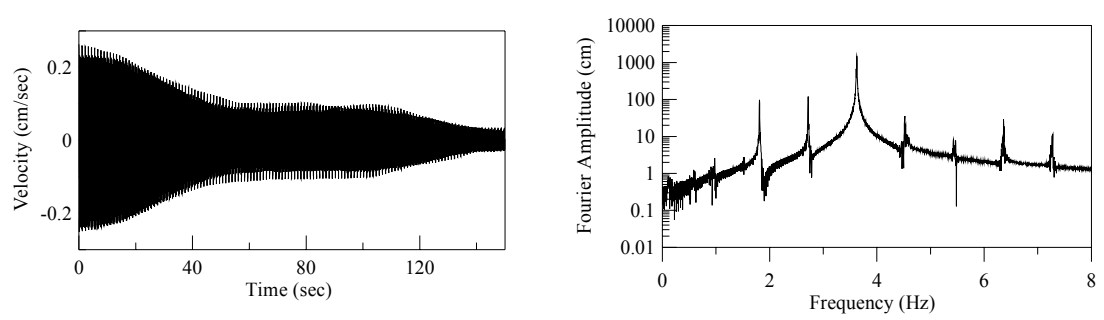

Figure 2: $\quad$ First round RD for Cable 33 of the Chi-Lu bridge.

\section{Mode separation and example}

Due to the inherited properties of uniformly separated modal frequencies and extremely small modal damping ratios, the interaction between any two modes of cable would be very limited. This feature was further exploited (Wu et al [4]) to develop a mode separation technique for effectively identifying the parameters of multiple modes. After Fourier transform is taken on the measured velocity time history, the modal frequencies of cable can be accurately determined from the associated Fourier amplitude spectrum. With these available modal frequencies to decide different frequency ranges for separation, the frequency response contributed by each mode of cable is then conveniently extracted in the frequency domain. In this research, the mid-points between any two adjacent modal frequencies are adopted to divide the frequency ranges for different modes. The corresponding time history for each separated mode is subsequently obtained by performing the inverse Fourier transform. Finally, the MRD method together with the ITD method can be independently applied on these individual modal responses to identify the dynamic parameters for each mode.

Cable 33 of the Chi-Lu bridge is again taken as an example. The parameters for the first 10 modes of this cable are aimed for identification. After the mode separation is performed as previously described, the MRD technique is applied on each separated modal time history with a cutting threshold $=0.8 \times$ standard deviation of the target time history and $T_{d}=$ half of the original time history for each round of RD. The MRD method is set to stop before the criterion of 
$N \geq 100$ cannot be guaranteed. In addition, the ITD technique is also utilized to identify the corresponding parameters after each round of RD. As discussed in Section 3, the time shifting for each mode is in consistent correspondence to $1 / 4$ of each modal period. The identified parameter values for each mode in all the $\mathrm{RD}$ rounds are arranged and listed in Table 2. Based on these results, it is obvious that the conventionally ambiguous modal damping ratios all reach stably convergent values, usually with very few rounds of $\mathrm{RD}$. This trend shows that not a lot of $\mathrm{RD}$ rounds are required to obtain satisfactory parameters from identification and also indicates the effective performance of the approach proposed in this study.

Table 2: $\quad$ Identified modal parameters of Cable L33 in each round of MRD.

\begin{tabular}{|c|c|c|c|c|c|c|c|c|c|c|}
\hline \multirow{2}{*}{ MRD } & \multicolumn{10}{|c|}{ Identified modal parameters } \\
\cline { 2 - 12 } & $\begin{array}{c}\omega_{1} \\
(\mathrm{~Hz})\end{array}$ & $\begin{array}{c}\xi_{1} \\
(\%)\end{array}$ & $\begin{array}{c}\omega_{2} \\
(\mathrm{~Hz})\end{array}$ & $\begin{array}{c}\xi_{2} \\
(\%)\end{array}$ & $\begin{array}{c}\omega_{3} \\
(\mathrm{~Hz})\end{array}$ & $\begin{array}{c}\xi_{3} \\
(\%)\end{array}$ & $\begin{array}{c}\omega_{4} \\
(\mathrm{~Hz})\end{array}$ & $\begin{array}{c}\xi_{4} \\
(\%)\end{array}$ & $\begin{array}{c}\omega_{5} \\
(\mathrm{~Hz})\end{array}$ & $\begin{array}{c}\xi_{5} \\
(\%)\end{array}$ \\
\hline 1st Round & 0.913 & 0.2061 & 1.808 & 0.0745 & 2.720 & 0.0994 & 3.627 & 0.1546 & 4.534 & 0.0630 \\
\hline 2nd Round & 0.914 & 0.2312 & 1.808 & 0.0559 & 2.720 & 0.0934 & 3.626 & 0.2007 & 4.534 & 0.0685 \\
\hline 3rd Round & 0.914 & 0.2349 & 1.807 & 0.0530 & 2.721 & 0.1003 & 3.625 & 0.2324 & 4.533 & 0.0717 \\
\hline \hline MRD & $\begin{array}{c}\omega_{6} \\
(\mathrm{~Hz})\end{array}$ & $\begin{array}{c}\xi_{6} \\
(\%)\end{array}$ & $\begin{array}{c}\omega_{7} \\
(\mathrm{~Hz})\end{array}$ & $\begin{array}{c}\xi_{7} \\
(\%)\end{array}$ & $\begin{array}{c}\omega_{8} \\
(\mathrm{~Hz})\end{array}$ & $\begin{array}{c}\xi_{8} \\
(\%)\end{array}$ & $\begin{array}{c}\omega_{9} \\
(\mathrm{~Hz})\end{array}$ & $\begin{array}{c}\xi_{9} \\
(\%)\end{array}$ & $\begin{array}{c}\omega_{10} \\
(\mathrm{~Hz})\end{array}$ & $\begin{array}{c}\xi_{10} \\
(\%)\end{array}$ \\
\hline 1st Round & 5.474 & 0.1302 & 6.365 & 0.0461 & 7.278 & 0.1075 & 8.221 & 0.0881 & 9.141 & 0.0480 \\
\hline 2nd Round & 5.473 & 0.1260 & 6.365 & 0.0444 & 7.279 & 0.1108 & 8.220 & 0.0696 & 9.141 & 0.0533 \\
\hline 3rd Round & 5.475 & 0.1140 & 6.365 & 0.0426 & 7.278 & 0.1141 & 8.220 & 0.0669 & 9.141 & 0.0560 \\
\hline 4th Round & $\cdot$ & $\cdot$ & 6.365 & 0.0431 & $\cdot$ & $\cdot$ & $\cdot$ & $\cdot$ & $\cdot$ & $\bullet$ \\
\hline
\end{tabular}

\section{References}

[1] Cole, H.A. Jr., Methods and apparatus for measuring the damping characteristics of a structure. United States Patent No. 3,620,069, 1971.

[2] Jeary, A.P., The description and measurement of nonlinear damping in structures. Journal of Wind Engineering and Industrial Aerodynamics, 59(2-3), pp. 103-114, 1996.

[3] Liao, C.-A., Modal parameter identification for both the cables and girders of cable-stayed bridges based on ambient vibration measurements, Master Thesis, National Yunlin University of Science \& Technology, 2007.

[4] Wu, W.-H., Liao, C.-A., and Chen, C.-C., A novel multiple random decrement method for modal parameter identification of cable-stayed bridge cables, International Operational Modal Analysis Conference 2007, paper no. 92, Copenhagen, Denmark, 2007. 